\title{
The MONS Sky
}

\author{
F.P. Pijpers \\ Theoretical Astrophysics Center, Aarhus University, Denmark
}

T.C. Teixeira

Institute of Physics and Astronomy, Aarhus University, Denmark

\begin{abstract}
The MONS project on the Rømer satellite aims at studying properties of stellar interiors through observations of oscillations in stellar luminosity and surface temperature. The main targets are stars exhibiting oscillations similar to those observed in the Sun, with very low amplitudes. During the planned two-year lifetime of the mission, around 20 such stars will be observed with the MONS Main Telescope. Additional data on variability of a large number of stars of a broad range of types may be obtained from the Star Trackers. In order to assist in planning of both ground-based science as well as mission science itself, various tools and databases have been or are being developed. These tools are/will be accessible through the MONS Information System (MIS) which can be queried through a WWW interface. One such tool is a clickable map of the full sky with on it the various fields around the designated main targets of MONS. A click on a field leads to information on the stars within those fields which can be of interest for asteroseismic studies.
\end{abstract}

\section{Providing observer support}

The MONS main camera will be pointed at 20 solar-like stars selected from the prioritized list in Table 1, for 30 days each. In Table 1. these stars are subdivided by column in various subclasses, and by row in levels of priority. As stated in the Rømer Science Mission Specification these levels are :

1. Very high priority, should definitely be observed.

2. High priority, should be observed.

Subclass $b$ targets are similar but may not be feasible ; should be observed if feasible.

3. Excellent target. A small subset of this group will be observed.

Subclass $b$ are similar but may not be feasible, should be added to priority 3 if feasible.

In addition to these stars are a group $\mathrm{A}(\lambda$ Ser, $\iota$ Per $)$ and a group B $\left(\pi^{3}\right.$ Ori, $\gamma$ Ser, $\gamma$ Lep, $\chi$ Dra, $\theta$ Per, $\iota$ Peg) of stars which require further study before a final selection can be made on which stars are to be put into the appropriate 
slots. There is also a group $\mathrm{C}$ of hotter stars $(\alpha$ Tri, $\gamma$ Dor, $\mu$ Vir, $\iota$ Leo, $\xi$ Gem, $\gamma$ Tuc, HR $4102, \alpha$ Oct) which are all priority 3 or $3 \mathrm{~b}$.

Table 1. The prioritized list of solar-like stars that have been selected as targets for the MONS main camera.

\begin{tabular}{|c|c|c|c|c|c|c|}
\hline $\begin{array}{l}\text { Priority } \\
\text { group }\end{array}$ & $\begin{array}{l}\text { Solar } \\
\text { Mass }\end{array}$ & $\begin{array}{c}\text { Lower } \\
\text { Mass }\end{array}$ & $\begin{array}{c}\text { Higher } \\
\text { Mass }\end{array}$ & $\begin{array}{l}\text { Metal } \\
\text { Poor }\end{array}$ & $\begin{array}{l}\text { Metal } \\
\text { Rich }\end{array}$ & $\begin{array}{l}\text { Rapid } \\
\text { rotator }\end{array}$ \\
\hline 1. & $\begin{array}{c}\alpha \text { Cen A } \\
\beta \text { Hyi }\end{array}$ & $\alpha$ Cen B & $\begin{array}{l}\eta \mathrm{Boo} \\
\alpha \mathrm{CMi}\end{array}$ & & & \\
\hline 2. & $\mu$ Her & & $\begin{array}{c}v \text { And } \\
\beta \text { Vir } \\
+1 \text { grp. B }\end{array}$ & $\nu$ Ind & $\delta \mathrm{Pav}$ & $\theta \mathrm{Boo}$ \\
\hline $2 \mathrm{~b}$. & & $\epsilon$ Eri & & HD 140283 & & \\
\hline 3. & $\begin{array}{c}\delta \text { Eri } \\
\eta \text { Cas } \\
+1 \text { grp. A }\end{array}$ & & $\begin{array}{c}\zeta \text { Her } \\
\alpha \text { For } \\
+3 \text { grp. B }\end{array}$ & $\gamma \mathrm{Pav}$ & & $\psi$ Сар \\
\hline $3 \mathrm{~b}$. & & $\begin{array}{c}\tau \text { Cet } \\
70 \mathrm{Oph} \\
36 \mathrm{Oph}\end{array}$ & & & & $\begin{array}{l}\kappa \text { Cet } \\
\chi^{1} \text { Ori }\end{array}$ \\
\hline
\end{tabular}

In addition to the main camera there are three instruments which can be used for science purposes : a forward pointing star tracker (FST) and a backward pointing star tracker (BST), as well as a field monitor (FM). All of these instruments can be used for asteroseismology. The limiting magnitude for the field monitor is much fainter and so there may even be extragalactic objects such as active galactic nuclei (AGN) which can be monitored with that instrument.

As part of any asteroseismic observing proposals to be submitted to the MONS science teams, it is required that the relevant basic stellar parameters of the target stars of the proposal be submitted to the MONS Information System (MIS). In some cases that may require preparatory ground-based observations. The MONS sky web site has been created in order to support observers who wish to make use of the instruments on Rømer, and to plan (auxiliary) observations. Through this site lists can be accessed of objects that lie within the respective fields of view of FST, BST, and FM when the MONS main telescope is pointed at any of the targets listed in Table 1. In the case of the FST and BST lists have been compiled of all stars down to a limiting magnitude of 9.5 in V and with a Hipparcos parallax determination better than 8 sigma. Some observed properties of these stars are included in these lists. As an example, if Strømgren photometry has already been carried out on a star the data is made available. In the case of the FM all known objects down to a limiting magnitude of 16 in $\mathrm{V}$ are listed. All lists are available as separate ASCII tables for each target field and for each instrument. The MONS sky can be accessed on the WWW through the MONS Information System : http://astro.ifa.au.dk/MIS.

Acknowledgments. The MONS sky data was compiled making extensive use of the SIMBAD database and of the VizieR catalogue access tool, operated at CDS, Strasbourg, France. The Theoretical Astrophysics Center (TAC) is created in collaboration between the Danish National Research Foundation, Copenhagen University and Aarhus University. 


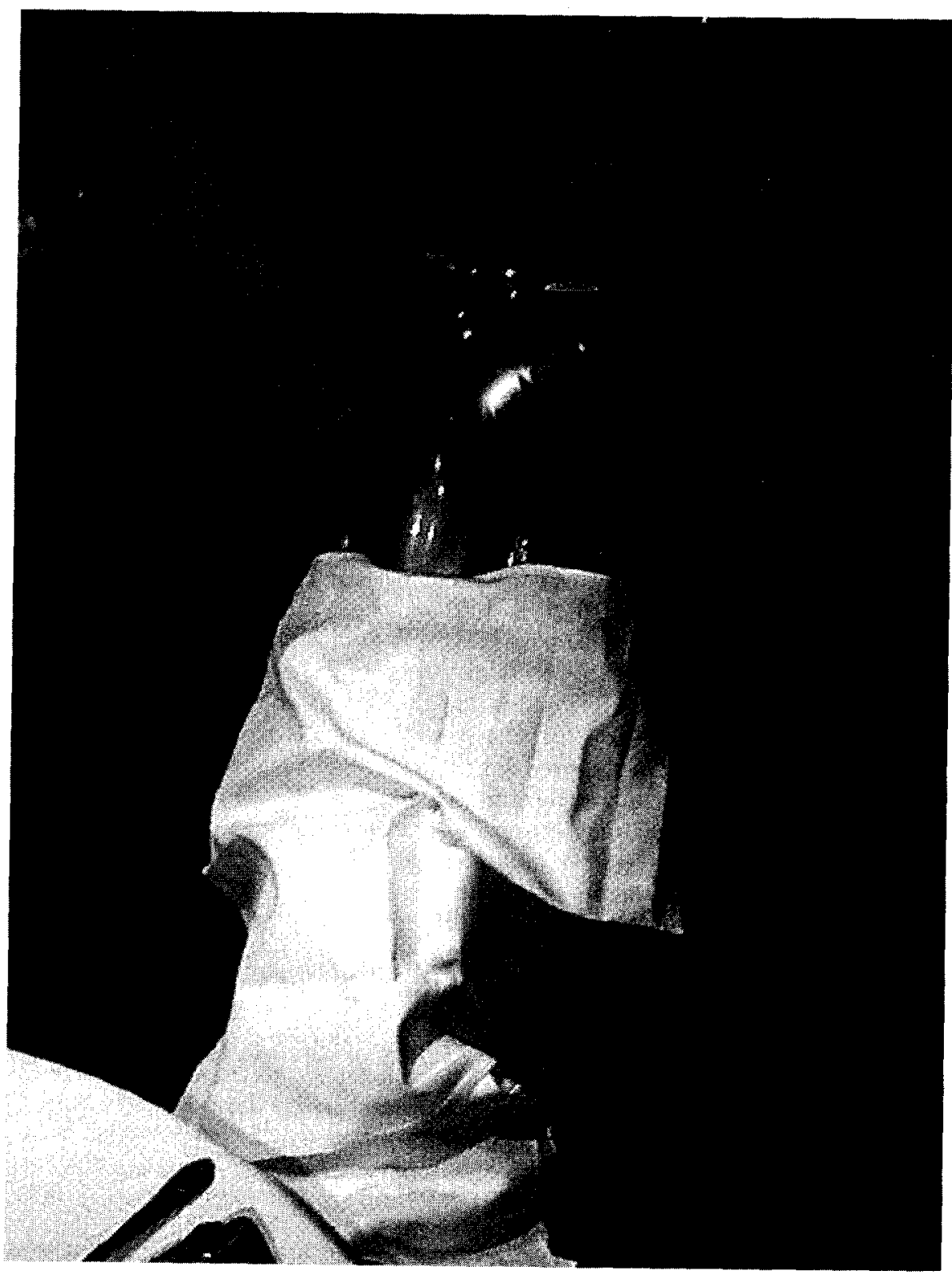

An alien all of a sudden dropped by in order to confuse Don Kurtz just before his closing lecture. But Don was not the least surprised as he had met this alien before... 